\section{Síndrome de muerte súbita del lactante: prevalencia y cambios en los últimos años en Chile}

\author{
PABLO E. BROCKMANN ${ }^{1}$, MARÍA ANGÉLICA OYARZÚN ${ }^{1}$, \\ LUIS VILLARROEL ${ }^{2}$, PABLO BERTRAND ${ }^{1}$
}

\section{Prevalence of sudden death syndrome among children}

Background: The sudden infant's death syndrome (SD) is the leading cause of death in children under one year. Despite advances in its study, the pathogenesis has not been yet fully elucidated. Aim: To assess the prevalence of SD in Chilean infants and its changes in recent years. Material and Methods: Review of birth and death databases of the Ministry of Health from 1997 to 2009. All cases diagnosed as SD, according to the International Classification of Diseases, 10th edition, were selected. A demographic analysis was performed and mortality rates for each year were calculated. Results: We identified 1442 cases of SD (847 males, 517 deaths at home). The median age of death was 2 months (0 to 11.0 months). Ninety six percent of deaths occurred in children aged $<6$ months. Mortality rate for $S D$ was $0.45 / 1000$ live births. There was a 23\% reduction between 1997 and 2009. When analyzing geographic distribution, more cases were found in the Southern latitudes of the country. Conclusions: The overall rate of SD in Chile is higher than in European countries and in North America. The observed decrease in cases over the years is still far from optimal.

(Rev Med Chile 2013; 141: 589-594).

Key words: Apnea; Childhood; Sudden infant death.

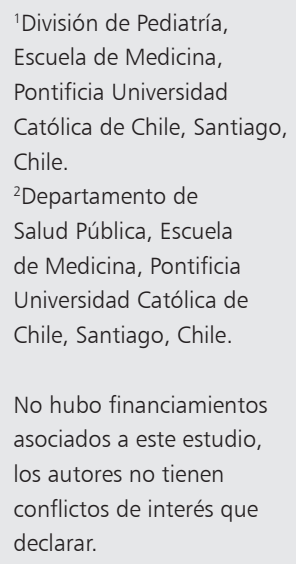

Recibido el 25 de julio de 2012, aceptado el 13 de diciembre de 2012.

Correspondencia a: Dr. Pablo E. Brockmann División de Pediatría, Escuela de Medicina, Pontificia Universidad Católica de Chile Lira 85, 5 piso, Santiago de Chile.

Teléfono: 56-2-3543767 E-mail: pbrockmann@med. puc.cl
L a muerte súbita del lactante (MS) es la principal causa de muerte en la población entre 1 mes y 1 año de vida ${ }^{1,2}$ en países desarrollados. Se define MS como el fallecimiento de un niño menor de un año, con historia, examen físico y autopsia normales, en las cuales no se ha podido establecer una causa definitiva de muerte conocida ${ }^{3}$. Debido a que el diagnóstico de MS implica descartar otras posibles causas de muerte, debe existir un protocolo preciso y se debe extremar la búsqueda de las probables patologías involucradas en este tipo de eventos. Todo lo anterior justifica la implementación de necropsias muy exigentes y la necesidad de hacer un diagnóstico diferencial correcto ${ }^{4}$.

La etiopatogenia de la MS aún no está clara. Una de las teorías más aceptadas se basa en la "triple teoría" 5 postulada por Wegdwood ya en $1972^{6}$ y ratificada en estudios posteriores por Kahn y $\operatorname{cols}^{7,8}$, la cual explica este fenómeno basado en tres factores: 1) riesgo innato del individuo; 2) edad de riesgo y 3) condición predisponente. Recientemente, se ha postulado como mecanismo principal involucrado en la MS una alteración en el umbral del despertar?.

Durante años la MS ha sido objetivo de múltiples estudios que han permitido mejorar el conocimiento y de sus factores relacionados. Se han establecidos campañas de prevención que han demostrado disminuir de manera importante la mortalidad relacionada a este síndrome. Es así como la campaña "back to sleep" (volver a dormir de espalda) realizada en países europeos y norteamericanos estimuló a gran parte de los padres a posicionar sus hijos en decúbito supino al dormir, consiguiendo de esta forma una significativa disminución de los casos de MS en este grupo ${ }^{10}$. 
La incidencia de MS es variable y depende de cada país. Series realizadas en los Estados Unidos de Norteamérica describen una frecuencia aproximada de 0,5/1.000 nacidos vivos; siendo esta frecuencia mayor en los niños de raza negra y menor en los de origen hispánico. En Argentina, una revisión publicada recientemente demostró una incidencia de 0,49 cada 1.000 nacidos vivos ${ }^{1}$. Este sería a nuestro saber el único representante de países latinoamericanos en ese estudio. En un grupo poblacional más acotado en el sur de Brasil se obtuvo una tasa de 0,55 cada 1.000 nacidos vivos ${ }^{11}$.

En Chile, pese a constituir una causa de muerte que debe ser de notificación obligatoria y encontrarse por ley eventualmente obligada a ser estudiadas por autopsias, se desconocen cifras exactas de su incidencia. Teniendo en cuenta la experiencia reportada en otros países, los cambios socioeconómicos de los últimos años y la significativa reducción de otras causas de morbimortalidad infantil en Chile, es planteable que la MS constituya uno de los principales problemas de salud pública a investigar en lactantes.

Por lo tanto, el objetivo del presente trabajo fue identificar la tasa de esta causa de muerte en lactantes chilenos y analizar la evolución y cambios a lo largo de los últimos años.

\section{Pacientes y Método}

Se revisaron las bases de datos de defunción del Ministerio de Salud de Chile, a través de la página del Departamento de Estadísticas e Información en Salud (www.deis.cl). Se incluyeron todas las bases de datos disponibles desde el año 1997 al 2009.

Se realizaron búsquedas electrónicas con buscadores en Excel, las cuales se confirmaron manualmente caso a caso. Se identificaron todos aquellos casos catalogados con la sigla "R95X" de la clasificación internacional de enfermedades, décima versión (CIE 10), correspondiente a la categoría de MS. Se filtraron manualmente en la base de datos en búsqueda de posibles errores en la base de datos original. Se excluyeron todos aquellos casos erróneamente catalogados como MS mayores de 1 año de vida al momento de fallecer, al no corresponder a la definición de la enfermedad.

Las tasas de mortalidad se calcularon en base a todos los casos detectados divididos por cada 1.000 nacidos vivos en cada año analizado. La tasa de nacimientos se obtuvo analizando las bases de datos del Departamento de Estadísticas e Información en Salud del Ministerio de Salud de Chile entre los años 1997 y 2009.

\section{Estadística}

Se realizó un análisis descriptivo del número de casos de MS por cada año, analizando de forma adicional los siguientes factores demográficos asociados a la presencia de MS: edad al momento de fallecer, género, edad gestacional al momento de nacer, presencia de prematurez (definida como una edad gestacional $<37$ semanas), mes del año al momento de fallecer y lugar de fallecimiento (hospital/domicilio). Estos análisis se realizaron con el programa SPSS versión 20.0

Adicionalmente, se evaluó la tendencia de las tasas de MS utilizando el modelo de Prais-Winsten (modelo autoregresivo de primer orden). Dado que la tasa tiene distribución Poisson, el modelo se ajustó usando ecuaciones de estimación generalizadas $(\mathrm{GEE})^{12}$. Para el análisis de la tendencia de las tasas por región, se usaron modelos Poisson de efectos mixtos. Para los análisis se usó el programa estadístico R (http://www.R-project.org) ${ }^{13}$. Se consideró significativo todo valor inferior o igual a 0,05 .

\section{Resultados}

Se identificaron un total de $\mathrm{n}=1.442$ casos de MS entre los años 1997-2009, correspondiendo $\mathrm{n}=847(59 \%)$ a varones. La razón varones a niñas fue de 1,42:1. La mediana (mínimo-máximo) de edad de fallecimiento de los lactantes fue de 2,0 meses ( 0 a 11,0 meses), $\mathrm{n}=346$ (24\%) presentaban el antecedente de prematurez. $81 \%$ de los casos fueron menores de 2 meses de edad. Noventa y seis por ciento falleció antes de los primeros 6 meses de vida. En $n=517$ (36\%) el fallecimiento fue en el domicilio, en $n=595(41,3 \%)$ en el hospital y en $\mathrm{n}=255(17,8 \%)$ en lugar no especificado (no hospitalario). En $n=75(5,2 \%)$ el lugar de fallecimiento no fue consignado. El antecedente de ser hijo(a) de madre soltera se obtuvo en $\mathrm{n}=899$ $(62,3 \%)$ de los casos. Las madres de $\mathrm{n}=83(5,8 \%)$ de los casos tenían cursado educación superior. El análisis por región geográfica demostró una mayor tasa en las regiones del sur del país (Figura 1). Los datos demográficos de todos los casos incluidos se presentan en la Tabla 1 y la distribución por edad en la Figura 2. 
El análisis dicotomizado por género demostró una menor edad al momento del fallecimiento en niñas que en varones (2,1 versus 2,4 meses respectivamente, $p=0,04)$. No hubo diferencias significativas respecto a la edad gestacional, antecedente de prematurez entre varones y niñas (Tabla 2).

Se demostró una distribución no homogénea de los casos de MS analizados, predominando durante los meses de invierno (Figura 3). La mayor cantidad de fallecimientos se registraron durante el mes de julio de cada año, sumando un total de $\mathrm{n}=190$ casos, seguido por el mes de junio con $\mathrm{n}=167$. La razón entre los casos identificados en invierno, comparados con los identificados en verano fue de 2,3: 1 (Figura 3).

La tasa de mortalidad promedio para los varones fue 0,27 y 0,19 en niñas. En ambos grupos se pudo observar un descenso en la tasas de morta-

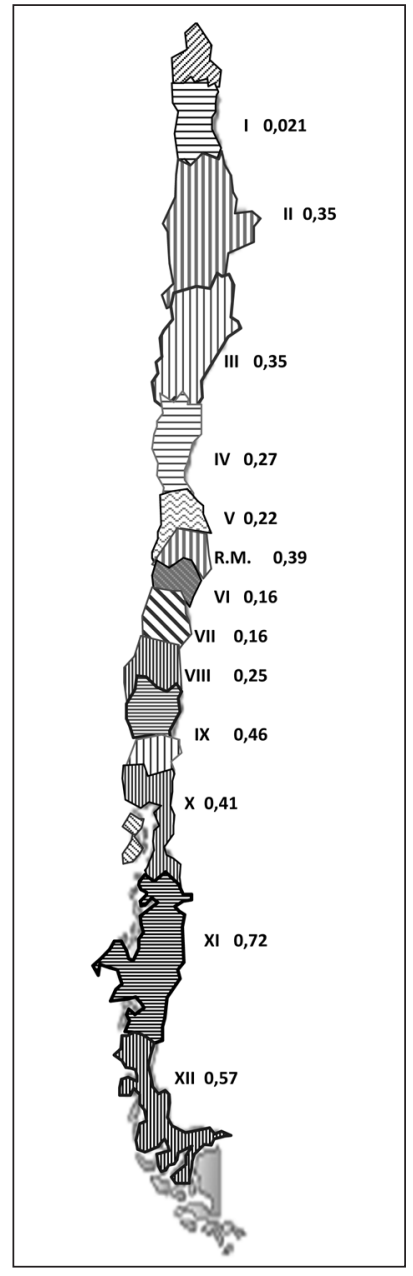

Figura 1. Tasa de muerte súbita del lactante según distribución geográfica. lidad a lo largo de los años analizados, disminuyendo $24 \%$ en varones y $17 \%$ en niñas.

La tasa nacional de mortalidad por MS global fue de 0,45 por cada 1.000 nacidos vivos y presenta durante el período estudiado un descenso significativo ( $p<0,001$ ), con una disminución de 5,3 casos en promedio al año, de acuerdo al modelo de Poisson.

El análisis por región usando modelos Poisson de efectos mixtos, arrojó los resultados mostrados en la Tabla 3. Los parámetros estimados con signo negativo indican una disminución de la tasa en el

Tabla 1. Características demográficas de los lactantes incluidos $(n=1.442)$

\begin{tabular}{|lc|}
\hline Característica & Resultado \\
\hline $\begin{array}{l}\text { Edad en meses al fallecer, mediana } \\
\text { (mínimo - máximo) }\end{array}$ & 2,0 \\
Varones, $\mathrm{n}(\%)$ & $(0-11,0)$ \\
\hline Antecedente de prematurez, n (\%) & $847(59)$ \\
\hline Fallecimiento en domicilio, n (\%) & $346(24)$ \\
\hline $\begin{array}{l}\text { Peso nacimiento gramos, } \\
\text { promedio } \pm \text { desviación estándar }\end{array}$ & $517(36)$ \\
\hline $\begin{array}{l}\text { Edad gestacional en semanas, } \\
\text { promedio } \pm \text { desviación estándar }\end{array}$ & $3.034 \pm 705$ \\
Madre soltera, n (\%) & $37,7 \pm 2,9$ \\
Nivel educacional materno ( $\mathrm{n}=1.333)$ & $899(62)$ \\
$\quad$ Básico, $\mathrm{n}(\%)$ & $555(38)$ \\
Medio, $\mathrm{n}(\%)$ & $695(51)$ \\
Superior, $\mathrm{n}(\%)$ & $83(6)$ \\
\hline
\end{tabular}

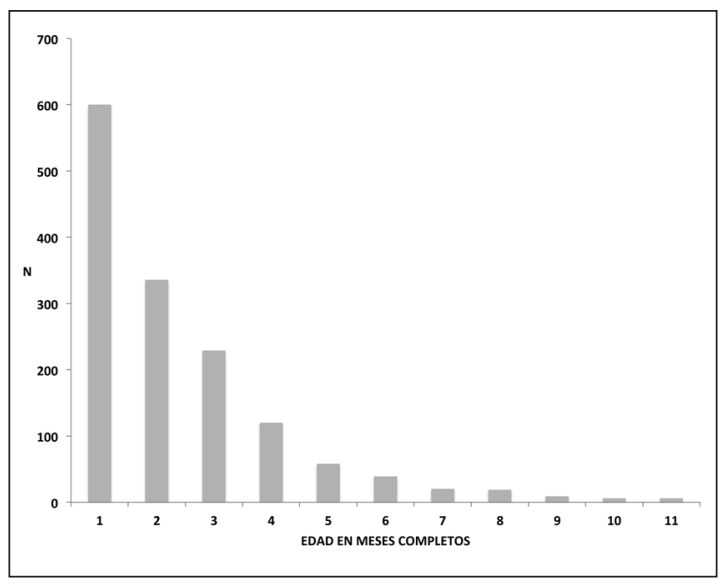

Figura 2. Distribución etaria de los lactantes al momento de fallecer $(n=1.442)$. 


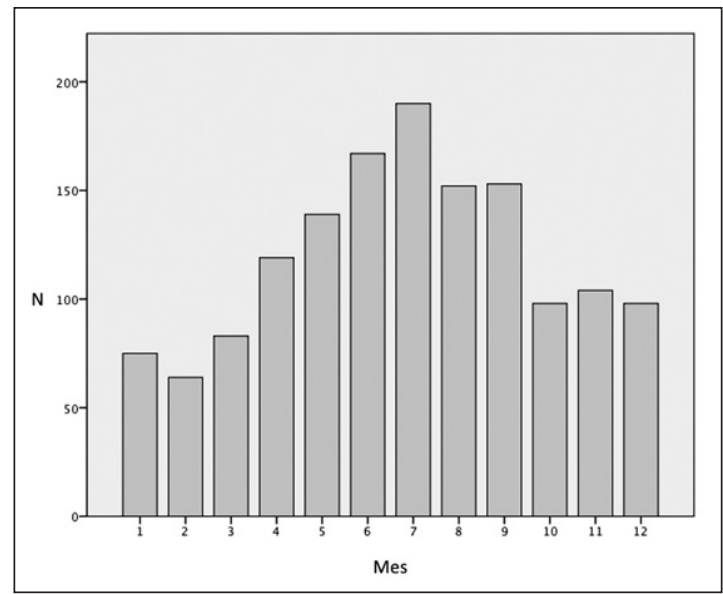

Figura 3. Distribución mensual de los casos de muerte súbita $(n=1.442)$.

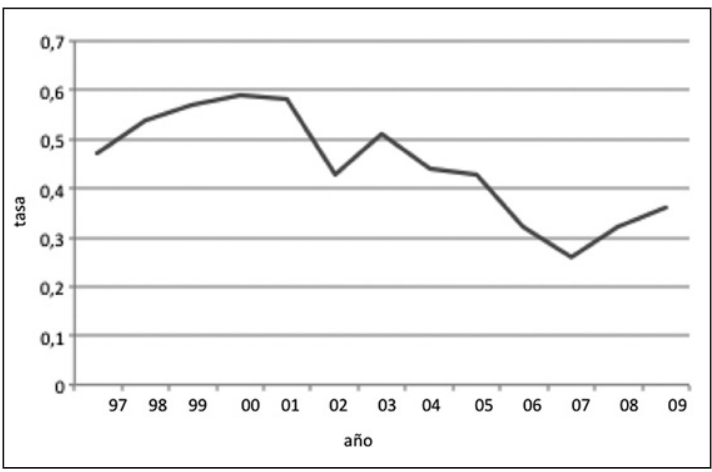

Figura 4. Tasa de mortalidad ( $n / 1000$ nacidos vivos) por muerte súbita del lactante según año.

tiempo y los parámetros estimados con signo positivo indican un aumento de la tasa. Las tasas en regiones $\mathrm{V}$, IX, X y RM muestran una disminución significativa en el tiempo. El resto de las regiones no muestra cambios significativos en la tasa de MS.

\section{Discusión}

El presente estudio demostró una tasa poblacional global de MS de 0,45 por cada 1.000 nacidos vivos en Chile. Por otro lado, revela una disminución en el número de casos desde el año 1997 al 2009. A nuestro saber, el presente estudio representa la primera publicación respecto a esta causa de muerte conocida en nuestro país.

La disminución progresiva de la tasa de fallecimiento por MS observada en el lactante es
Tabla 3. Evolución de la tasa de MS según región del país

\begin{tabular}{|ccr|}
\hline Región & $\begin{array}{c}\text { Parámetro Poisson } \pm \\
\text { error estándar }\end{array}$ & Valor p \\
I & $-0,055 \pm 0,048$ & 0,256 \\
II & $-0,024 \pm 0,038$ & 0,513 \\
III & $-0,049 \pm 0,037$ & 0,182 \\
IV & $0,051 \pm 0,039$ & 0,187 \\
V & $-0,099 \pm 0,022$ & $<\mathbf{0 , 0 0 1}$ \\
VI & $-0,059 \pm 0,044$ & 0,183 \\
VII & $0,009 \pm 0,037$ & 0,797 \\
VIII & $0,048 \pm 0,027$ & 0,074 \\
IX & $-0,109 \pm 0,028$ & $<\mathbf{0 , 0 0 1}$ \\
X & $-0,064 \pm 0,023$ & $\mathbf{0 , 0 0 3}$ \\
XI & $-0,077 \pm 0,042$ & 0,070 \\
XII & $-0,014 \pm 0,050$ & 0,777 \\
RM & $-0,055 \pm 0,011$ & $<\mathbf{0 , 0 0 1}$ \\
\hline
\end{tabular}

* Modelos Poisson de efectos mixtos: los parámetros estimados con signo negativo indican una disminución de la tasa en el tiempo y los parámetros estimados con signo positivo indican un aumento de la tasa.

concordante con lo sucedido en otros países desde la década del 1990. Una revisión reciente reunió los datos de mortalidad por MS de 13 países ${ }^{1}$, demostrando una disminución generalizada, cuyo rango fluctuaba entre $40 \%$ y $87 \%$ de reducción. La reducción demostrada en nuestro país, sin embargo, fue menor. Las razones de esta menor disminución pueden ser varias, sin embargo, este hecho es preocupante. La mayoría de los países han realizado campañas educativas desde la década 1990-99 para educar a la población sobre la MS y su prevención. Una de estas campañas fue la ya mencionada "back to sleep", por la cual se logró reducir significativamente la MS una vez instaurada esta política de salud pública ${ }^{1}$. En Chile, pese a que existe conocimiento general sobre la recomendación acerca de dormir en decúbito supino, aún no se han puesto en marcha políticas de salud masivas.

La tasa global demostrada en Chile es similar al promedio de los países desarrollados antes de las campañas de prevención de MS en los $1990^{2}$. Si bien esta tasa es comparable a las publicadas por países vecinos como Argentina y Brasil (ambas al- 
rededor de los 0,5 por cada 1.000 nacidos vivos ${ }^{1,11}$, todavía es muy distante a las tasas de publicadas en los Países Bajos o Japón con 0,1 y 0,16 por cada 1.000 nacidos vivos, respectivamente ${ }^{14,15}$.

$\mathrm{Al}$ igual que lo demostrado previamente en el extranjero, se pudo reconocer una serie de factores de riesgo para MS. La edad de fallecimiento fue en la mayor parte de los casos (> 80\%) antes de los 2 meses de vida. La edad sería uno de los factores descritos en la "triple teoría" y otros factores de riesgo clínicos descritos por autores como Kahn y $\operatorname{cols}^{16}$. La triple teoría postula que la MS se debe a: una vulnerabilidad innata, edad y noxa exógena. Dentro de los factores descritos como parte de esta vulnerabilidad innata están la alteración en el despertar ante ciertos estímulos como la hipoxemia e hipercarbia ${ }^{17}$. El deficiente control del despertar estaría menos desarrollado en algunos sujetos más susceptibles, en especial en niño(a)s nacidos de pretérmino ${ }^{18}$. Esta situación de particular vulnerabilidad pudiera verse agravada por factores externos como: dormir en posición prona, infecciones respiratorias, tabaquismo en el hogar, sobre abrigo y colecho ${ }^{19}$. En nuestra serie, casi un cuarto de los casos presentaba el factor de riesgo de haber nacido pre término. Esto coincide tanto con los datos extranjeros, como con la hipótesis que la mayor inmadurez de los niños prematuros sería un factor de riesgo para la MS ${ }^{20,21}$. Además, destacó, al igual que en las publicaciones previas, una mayor tasa en varones que en niñas. La razón por la cual los varones tendrían mayor riesgo de desarrollar MS es desconocida. Por otro lado, destacó una alta presencia de parámetros sugerentes de un nivel socioeconómico bajo, como el nivel educacional materno. Estos datos corroboran lo encontrado en estudios internacionales que han asociado el nivel socioeconómico bajo y hacinamiento a la $\mathrm{MS}^{5}$.

Una de las características más interesantes del presente estudio fue el hallazgo de un incremento significativo a más del doble de la tasa de MS durante el invierno, en comparación con los meses de verano. Interesantemente, agentes infecciosos como el virus sincicial se han asociado a la presencia de apneas en lactantes ${ }^{22,23}$. Planteamos la hipótesis de que un número de los lactantes fallecidos durante los meses de invierno pudiere haber estado infectado por alguno de estos agentes. Además, se pudo observar una distribución geográfica de los casos de MS, mayor específicamente en las regiones IX, X, XI y XII. Las razones de esta diferencia geográfica pudieran ser múltiples, como por ejemplo una distinta prevalencia de enfermedades respiratorias, distintos hábitos de sueño, o bien una mayor rigurosidad en la notificación de la enfermedad. Sin embargo, estas hipótesis deben de ser confirmadas en estudios futuros.

El presente estudio presenta la gran ventaja de mostrar una tasa de mortalidad basada en datos nacionales, es decir, reunió todos los datos emanados de los certificados de defunción que se han emitido durante los años analizados. El número de casos encontrados $(>1.000)$ posiciona a este estudio dentro de los que más casos han incluido, siendo comparable en este sentido a estudios realizados en Europa o Norteamérica ${ }^{1}$. La tasa poblacional encontrada nos permite conocer de forma real cuál es el impacto de la MS en toda la población chilena. Sin embargo, dentro de las posibles limitaciones de este tipo de estudios está la pérdida de algunos casos por haber sido otro el diagnóstico inscrito en el certificado de defunción. Este fenómeno es propio de la mayoría de las revisiones, ya que la MS reviste una serie de problemas diagnósticos. El principal problema es la limitación de las autopsias. En la revisión previamente citada, la definición de MS varió dependiendo del país, no en todos los casos estaba claro si se había realizado una autopsia y una investigación exhaustiva del fallecimiento, como lo recomiendan las definiciones internacionales ${ }^{3,4}$. Por otra parte, proponemos utilizar definir como MS a todos los fallecimientos no explicados entre los 0 a 365 días de vida. La gran mayoría de los países que han publicado sus datos han utilizado esta definición, sin embargo, existe un grupo de países que la definen entre los 7 a 365 días de vida ${ }^{1}$. Por otro lado, los estudios epidemiológicos como el presente permiten identificar una serie de factores demográficos asociados a la MS.

Queremos destacar que el presente estudio no puede pretender esclarecer el complejo fenómeno de la MS ni probar causalidad. En el análisis no se contó con la descripción de otros factores asociados, en especial la presencia de tabaquismo o la posición al dormir del(a) niño(a) fallecido(a). Sin embargo, creemos que los resultados encontrados dan pie para poner en marcha una caracterización sistemática de esta patología a nivel nacional.

A pesar de haber demostrado una disminución de la tasa de MS a lo largo del tiempo en Chile, la tasa encontrada y la velocidad de descenso de ésta son preocupantes. Este hecho debe alertar- 
nos sobre la necesidad de buscar activamente la prevención de los factores de riesgo corregibles asociados a la MS.

\section{Conclusiones}

El presente estudio logró demostrar una tasa global de MS en Chile de 0,45/1.000 nacidos vivos. Si bien se observó una disminución importante en el número de casos entre los años 1997 al 2009, la tasa global de MS dista aún de ser comparable a países en los cuales se han puesto en marcha campañas poblacionales para su prevención. Nuestros datos plantean la necesidad de un registro sistemático de todos los factores de riesgo asociados a los casos registrados y de la puesta en marcha de campañas para la prevención de la MS.

Agradecimientos: El equipo de trabajo agradece al Departamento de Estadísticas e Información en Salud (www.deis.cl) del Ministerio de Salud de Chile por facilitar sus bases de datos.

\section{Referencias}

1. Hauck FR, Tanabe KO. International trends in sudden infant death syndrome: stabilization of rates requires further action. Pediatrics 2008; 122 (3): 660-6.

2. Leach CE, Blair PS, Fleming PJ, Smith IJ, Platt MW, Berry PJ, et al. Epidemiology of SIDS and explained sudden infant deaths. CESDI SUDI Research Group. Pediatrics 1999; 104 (4): e43.

3. Beckwith JB. Defining the sudden infant death syndrome. Arch Pediatr Adolesc Med 2003; 157 (3): 286-90.

4. Willinger M, James LS, Catz C. Defining the sudden infant death syndrome (SIDS): deliberations of an expert panel convened by the National Institute of Child Health and Human Development. Pediatr Pathol 1991; 11 (5): 677-84.

5. Guntheroth WG, Spiers PS. The triple risk hypotheses in sudden infant death syndrome. Pediatrics 2002; 110 (5): e64.

6. Wedgwood R, editor. Review of USA experience.Sudden and Unexpected Death in Infancy (Cot Deaths). Bristol: Wright; 1972.

7. Kahn A. [Sudden and unexplained infant death]. Bull Mem Acad R Med Belg 1983; 138 (3): 225-35.

8. Kahn A, Groswasser J, Rebuffat E, Sottiaux M, Blum D, Foerster M, et al. Sleep and cardiorespiratory characteristics of infant victims of sudden death: a prospective case-control study. Sleep 1992; 15 (4): 287-92.
9. Franco P, Szliwowski H, Dramaix M, Kahn A. Decreased autonomic responses to obstructive sleep events in future victims of sudden infant death syndrome. Pediatric Research 1999; 46 (1): 33-9.

10. Ball HL, Volpe LE. Sudden Infant Death Syndrome (SIDS) risk reduction and infant sleep location - Moving the discussion forward. Soc Sci Med 2012.

11. Peres LC. Sudden unexpected infant death syndrome in Ribeirao Preto, Brazil. Sao Paulo Med J 1998; 116 (5): 1803-7.

12. Hedeker D, Gibbons R. Longitudinal data analysis. John Wiley and sons. 2006; New Jersey.

13. Team RDCR. A language and environment for statistical computing. R. Foundation for Statistical Computing, Vienna, Austria 2012

14. Sawaguchi T, Namiki M. Recent trend of the incidence of sudden infant death syndrome in Japan. Early Hum Dev 2003; 75 Suppl: S175-9.

15. Carpenter RG, Irgens LM, Blair PS, England PD, Fleming $\mathrm{P}$, Huber J, et al. Sudden unexplained infant death in 20 regions in Europe: case control study. Lancet 2004; 363 (9404): 185-91.

16. Kahn A, Blum D, Hennart P, Sellens C, Samson-Dollfus D, Tayot J, et al. A critical comparison of the history of sudden-death infants and infants hospitalised for nearmiss for SIDS. Eur J Pediatr 1984; 143 (2): 103-7.

17. Franco P, Szliwowski H, Dramaix M, Kahn A. Decreased autonomic responses to obstructive sleep events in future victims of sudden infant death syndrome. Pediatr Res 1999; 46 (1): 33-9.

18. Horne RS, Bandopadhayay P, Vitkovic J, Cranage SM, Adamson TM. Effects of age and sleeping position on arousal from sleep in preterm infants. Sleep 2002; 25 (7): 746-50.

19. Horne RS, Ferens D, Watts AM, Vitkovic J, Lacey B, Andrew S, et al. Effects of maternal tobacco smoking, sleeping position, and sleep state on arousal in healthy term infants. Arch Dis Child Fetal Neonatal Ed 2002; 87 (2): F100-5.

20. Horne RS, Andrew S, Mitchell K, Sly DJ, Cranage SM, Chau B, et al. Apnoea of prematurity and arousal from sleep. Early Hum Dev 2001; 61 (2): 119-33.

21. Horne RS, Sly DJ, Cranage SM, Chau B, Adamson TM. Effects of prematurity on arousal from sleep in the newborn infant. Pediatr Res 2000; 47 (4 Pt 1): 468-74.

22. Lindgren C, Jing L, Graham B, Grogaard J, Sundell H. Respiratory syncytial virus infection reinforces reflex apnea in young lambs. Pediatr Res 1992; 31 (4 Pt 1): 381-5.

23. Ralston S, Hill V. Incidence of apnea in infants hospitalized with respiratory syncytial virus bronchiolitis: a systematic review. J Pediatr 2009; 155 (5): 728-33. 